\title{
Relações entre propriedades de rigidez para distintas solicitações mecânicas visando projetos de estruturas de madeira
}

\author{
Relationship between stiffness properties for different \\ mechanical demands in timber structures design projects
}

\author{
João Paulo Boff Almeida \\ Natalia Garcia Couto \\ Vinicius Borges de Moura Aquino \\ Anderson Renato Vobornik Wolenski \\ Rodrigo Guerra Peixoto \\ André Luis Christoforo \\ Francisco Antonio Rocco Lahr
}

\section{Resumo}

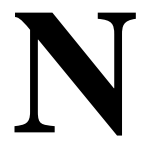

o Brasil, o documento normativo NBR 7190 (ABNT, 1997) estabelece os métodos de ensaio para obtenção das propriedades físico-mecânicas da madeira. Na impossibilidade da determinação dos resultados experimentais, a norma estabelece relações entre propriedades. Porém as relações entre as propriedades de rigidez apresentadas foram determinadas com base no antigo referencial normativo MB-26:1940

1 João Paulo Boff Almeida 1 Universidade Federal de São Carlos São Carlos - SP - Brasil

${ }^{2}$ Natalia Garcia Couto 2Universidade Federal de São Carlos São Carlos - SP - Brasil

${ }^{3}$ Vinicius Borges de Moura Aquino

${ }^{3}$ Universidade Federal do Sul e Sudeste do Pará Santana do Araguaia - PA - Brasil

${ }^{4}$ Anderson Renato Vobornik Wolenski ${ }^{4}$ Instituto Federal de Santa Catarina São Carlos - SC - Brasil

${ }^{5}$ Rodrigo Guerra Peixoto ${ }^{5}$ Universidade Federal de Minas Gerais Belo Horizonte - MG - Brasil

${ }^{6}$ André Luis Christoforo ${ }^{6}$ Universidade Federal de São Carlos São Carlos - SP- Brasil

${ }^{7}$ Francisco Antonio Rocco Lahr 7Universidade de São Paulo São Carlos - SP - Brasil

Recebido em 26/02/19 Aceito em 07/08/19
(NBR 6230), que considerava outro modelo de cálculo e não ao utilizado pela norma brasileira. Nesse contexto, este trabalho objetivou investigar a equivalência entre os módulos de elasticidade na flexão $\left(\mathrm{E}_{\mathrm{M}}\right)$, compressão $\left(\mathrm{E}_{\mathrm{c} 0}\right)$ e tração $\left(\mathrm{E}_{\mathrm{t} 0}\right)$ paralela às fibras de dez espécies de madeiras tropicais, assim como de estabelecer correlações adequadas entre tais propriedades em caso de não equivalência. Os resultados da ANOVA acusaram que $\mathrm{E}_{\mathrm{c} 0}$ e $\mathrm{E}_{\mathrm{t} 0} \mathrm{são}$ estatisticamente equivalentes, o mesmo não ocorreu entre $\mathrm{E}_{\mathrm{M}}$ e $0,90 \cdot \mathrm{E}_{\mathrm{c} 0}$, implicando a determinação do coeficiente ótimo de 0,98 pelo método dos mínimos quadrados. Tal coeficiente mostrou ser $8,89 \%$ superior ao apresentado pela norma brasileira.

Palavras-chave: Relações entre propriedades. Madeiras tropicais. Rigidez. Teoria de vigas.

\section{Abstract}

In Brazil, the Brazilian Standard NBR 7190 (ABNT, 1997) establishes testing methods to obtaining the physical and mechanical properties wood. With the impossibility of determining the experimental results, the Brazilian Code establishes relations among the properties. However, the relations between the stiffness properties are based on the older normative standard MB-26: 1940 (Brazilian Standard NBR 6230), which condisidered another calculation model rather than the one used by the Brazilian standard. This research aimed to investigate the equivalence between the conventional modulus of elasticity in static bending tests $\left(E_{M}\right)$, with compression parallel to the fibres $\left(E_{c 0}\right)$ and tension parallel to the fibres $\left(E_{t 0}\right)$ of ten species of tropical wood, as well as to establish relations between these properties, in case they are not equivalent. The ANOVA results showed that $E_{c 0}$ and $E_{t 0}$ were statistically equivalent, and the relation between $E_{M}$ and $0.90 \cdot E_{c 0}$ was not statistically equivalent, implying the determination of an ideal coefficient equal to 0.98 by the least squares method. This coefficient was $8.89 \%$ higher than the Brazilian standard.

Keywords: Relations between properties. Hardwood. Stiffness. Beams theory. 


\section{Introdução}

A madeira, material natural renovável e de fácil obtenção, é a matéria-prima mais antiga adotada em construções. Devido à sua ampla disponibilidade na natureza e relativa facilidade de manuseio, tem sido usada pela humanidade ao longo da história, atendendo atividades humanas habituais, como superar obstáculos, transporte, moradias, entre outros (CHRISTOFORO et al., 2013a; ARAUJO et al., 2016; WIERUSZEWSKI; MAZELA, 2017; ALMEIDA et al., 2018a).

Juntamente com o aço e o concreto armado, a madeira é atualmente um dos três materiais estruturais utilizados na construção de grandes estruturas. No que diz respeito à resistência à compressão, a madeira apresenta valor semelhante ao concreto e ambos os materiais possuem resistência inferior ao aço; no entanto, a madeira possui baixa densidade quando comparada a esses outros materiais estruturais, resultando em uma excelente relação entre resistência e densidade (RAMAGE et al., 2017). Segundo Calil Jr. e Dias (1997), essa relação é aproximadamente três vezes maior que para o aço e dez vezes maior que para o concreto, o que evidencia a madeira como um material estrutural eficiente, sendo de uso inteligente em estruturas nas quais uma alta proporção das cargas a ser resistida é seu peso próprio, como em estruturas de cobertura, algumas pontes e edifícios altos (RAMAGE et al., 2017).

Para a maioria dos países da América do Norte e Europa, casas de madeira são a solução habitacional mais comum, econômica e prática (ARAUJO et al., 2016). No Reino Unido aproximadamente $29 \%$ das novas residências são em madeira, na Escócia esse percentual é na ordem de 83\%, na Inglaterra 23\%, e na Irlanda do Norte 17\% (ARAUJO et al., 2018). Na Áustria, construções em wood frame representam cerca de $40 \%$ do total de edificações unifamiliares (HURMEKOSKI; JONSSON; NORD, 2015), no Japão 45\%, na Nova Zelândia tem-se 85\%, chegando aos impressionantes $90 \%$ na América do Norte (MAHAPATRA; GUSTAVSSON; HEMSTRÖM, 2012).

Com 8.715 espécies do total de 60.065 de árvores registradas em todo o mundo, o Brasil é o país com maior diversidade de espécies do planeta (BEECH et al., 2017). Apesar de ter uma área de cobertura florestal nativa de $60,7 \%$ do território nacional, e do evidente potencial florestal, o aproveitamento dos recursos naturais madeireiros é insignificante, em função do potencial que representa (VIDAL et al., 2015). Têm-se, como exemplo, as construções em madeira que ainda sofrem alguns preconceitos devido a questões culturais remanescentes desde a colonização, a escassez de mão de obra qualificada e o desconhecimento das espécies e suas propriedades. Tais fatores induzem o uso inadequado da madeira, com construções com baixa vida útil e que sucumbem às vantagens deste material (PEDRESCHI; GOMES; MENDES, 2005).

Para a utilização racional da madeira nas mais diversas finalidades, torna-se necessário o conhecimento de suas propriedades físicas, químicas, mecânicas e anatômicas (ALMEIDA et al., 2014, 2018b). Dentre as propriedades requisitadas para o dimensionamento de uma estrutura, destaca-se o módulo de elasticidade. Em um projeto de vigas, assim como outros elementos estruturais, o conhecimento dos módulos de elasticidade longitudinal (E) e transversal (G) é de suma importância (CHRISTOFORO et al., 2013b).

No Brasil, a Associação Brasileira de Normas Técnicas (ABNT) através do seu documento normativo n. 7.190 de 1997 (ABNT, 1997), intitulado de "Projeto de Estruturas de Madeira", estabelece as premissas e os métodos de cálculo para dimensionamento das estruturas em madeira, assim como os métodos de ensaios para caracterização completa das propriedades de resistência e rigidez da madeira.

Para a obtenção das propriedades das espécies de madeira, a norma exige procedimentos realizados em laboratórios especializados e com equipamentos de alto custo (DIAS e LAHR, 2004). Considerando a impossibilidade da obtenção dessas propriedades via experimentação, a norma brasileira propõe algumas relações entre propriedades mecânicas.

Dentre tais relações, a NBR 7.190 (Item 6.3.4 (ABNT, 1997)) estabelece que o valor médio do módulo de elasticidade na compressão paralela às fibras $\left(\mathrm{E}_{\mathrm{c} 0}\right)$ assume o mesmo valor do módulo de elasticidade na tração paralela às fibras $\left(E_{t 0}\right)\left[E_{c 0}=E_{t 0}\right]$, sem fazer distinção entre folhosas e coníferas. Para as folhosas, que são usualmente utilizadas em projetos estruturais devido a sua alta resistência, o mesmo referencial normativo permite, na impossibilidade do ensaio de compressão simples, a determinação do $\mathrm{E}_{\mathrm{c} 0}$ por meio do ensaio de flexão estática, considerando que o módulo de elasticidade convencional na flexão $\left(\mathrm{E}_{\mathrm{M}}\right)$ é igual a $0,90(90 \%)$ do módulo de elasticidade na compressão paralela às fibras $\left[\mathrm{E}_{\mathrm{M}}=0,90 \cdot \mathrm{E}_{\mathrm{c} 0}\right]$.

Segundo a norma brasileira, para obtenção das propriedades de resistência e rigidez da madeira na flexão, os corpos de prova devem possuir as dimensões $5 \mathrm{~cm} \times 5 \mathrm{~cm} \times 115 \mathrm{~cm}$, respeitando-se a relação entre o vão do ensaio de flexão $(L)$ e a altura da seção $(h)$ igual a 21 . Tal relação, obtida por Lahr (1983), utiliza-se do ensaio de flexão estática a três pontos em peças de madeira serrada. Os valores do módulo de elasticidade

26 Almeida, J. P. B.; Couto, N. G.; Aquino, V. B. de M.; Wolenski, A. R. V.; Peixoto, R. G.; Christoforo, A. L.; Lahr, F. A. R. 
obtidos com o auxílio da Equação 1, que contabiliza apenas a existência dos momentos fletores (sendo $\delta$ o deslocamento no ponto médio, $F$ o carregamento aplicado no meio do vão, $L$ o vão entre os apoios, $E_{M} \mathrm{O}$ módulo de elasticidade na flexão e $I$ o momento de inércia da seção transversal), apresentam ser equivalentes (convergentes) para relações $L / h \geq 21$, e distintos para relações inferiores. Esse resultado garante que os esforços cisalhantes presentes no ensaio de flexão possam ser desprezados na determinação dos deslocamentos, validando o modelo de cálculo (teoria de vigas de Euller Bernoulli) utilizado pela norma brasileira NBR 7190 (ABNT, 1997) e por outros documentos normativos para a determinação do módulo de elasticidade na flexão (ZANGIÁCOMO; CHRISTOFORO; LAHR, 2014).

$$
E_{M}=\frac{F \cdot L^{3}}{48 \cdot \delta \cdot I}
$$

A NBR 7190 (ABNT, 1997) substituiu e cancelou o antigo documento normativo MB-26:1940 (NBR 6230), o qual preconizava que os corpos de prova destinados à obtenção das propriedades de resistência e rigidez da madeira na flexão deveriam ter dimensões de $2 \mathrm{~cm} \times 2 \mathrm{~cm} \times 30 \mathrm{~cm}$, implicando a relação $L / h<21$. Os resultados obtidos conforme a metodologia de ensaio proposta pela MB-26:1940 (NBR 6230) condicionaram a relação de equivalência entre o módulo de elasticidade na flexão e o módulo de elasticidade na compressão paralela às fibras $\left(\mathrm{E}_{\mathrm{M}}=0,90 \cdot \mathrm{E}_{\mathrm{c} 0}\right)$, atualmente presente na NBR 7190 (Item 6.3 .4 (ABNT, 1997)). Com a mudança das dimensões dos corpos de prova pela NBR 7190 (ABNT, 1997), implicando a relação $L / h \geq 21$, fazem-se necessários novos estudos sobre a relação de equivalência entre tais propriedades.

Notadamente, tais propriedades são de extrema importância para o dimensionamento estrutural. Considerando as dificuldades associadas à caracterização completa de madeiras, juntamente com a possibilidade de relações entre propriedades, esta pesquisa objetiva investigar as relações entre os módulos de elasticidade na flexão, compressão e tração paralela às fibras das madeiras folhosas, com resultados em potencial para serem incorporados nas normas brasileiras, em especial na NBR 7190 (ABNT, 1997), atualmente em revisão.

\section{Materiais e métodos}

As madeiras de cambará rosa (Erisma sp.), cedro (Cedrella sp.), copaíba (Copaiba resinifera), canafístula (Cassia ferruginea), oiticica amarela (Clarisia racemosa), angelim araroba (Vataireopsis aroroba), louro verde (Ocotea sp.), guaiçara (Luetzelburgia sp.), cedroarana (Cedrelinga cateniformis) e maçaranduba (Manilkara sp.) foram devidamente estocadas e ensaiadas nas dependências do Laboratório de Madeiras e de Estruturas de Madeira (LaMEM) pertencente ao Departamento de Engenharia de Estruturas (SET), da Escola de Engenharia de São Carlos (EESC), da Universidade de São Paulo (USP), responsável por conduzir os ensaios experimentais com o rigor requerido pela norma brasileira.

Para tanto, as 10 espécies pesquisadas foram ensaiadas com a umidade de equilíbrio de $12 \%$, como estabelecido na NBR 7190 (p. 47, Anexo B, (ABNT, 1997)), com experimentos conforme premissas e métodos de ensaio e de cálculo deste documento normativo. A Figura 1 esquematiza a forma de extração e dimensões dos corpos de prova, conforme preconiza essa norma.

Figura 1 - Esquema de extração e dimensões dos corpos de prova para os ensaios dos módulos de elasticidade na flexão, compressão e tração paralela às fibras
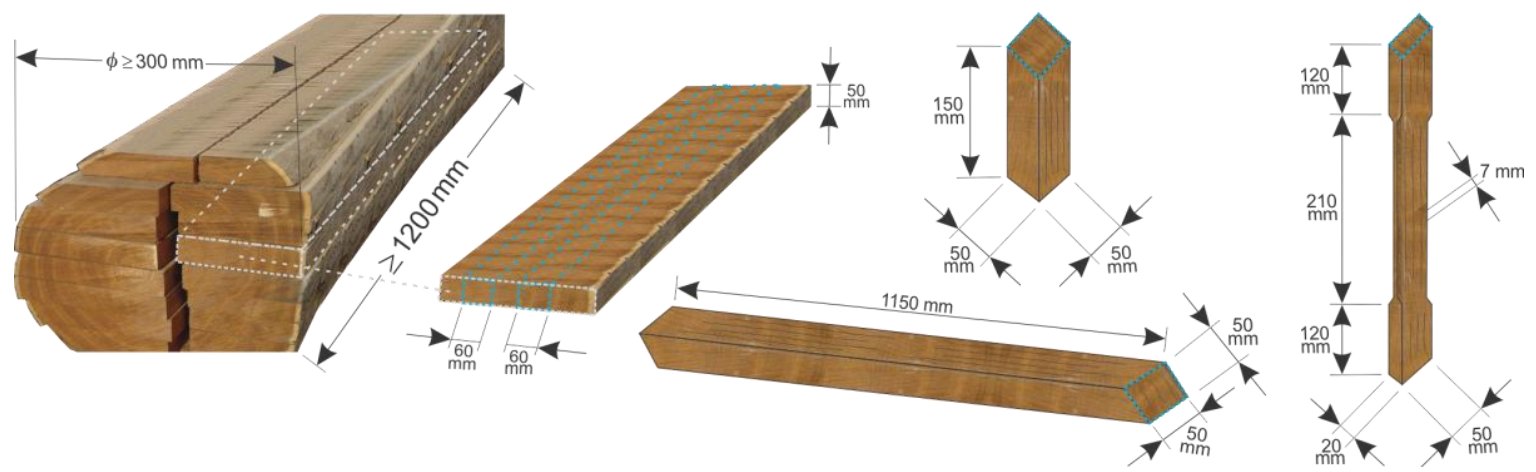
As espécies de madeiras foram categorizadas em classes de resistência para o grupo das folhosas de acordo com o valor característico (Equação 2) obtido do ensaio de compressão paralela às fibras (NBR 7190, 1997).

$$
f_{c 0, k}=\left(2 \cdot \frac{f_{1}+f_{2}+f_{3}+\ldots+f_{(n / 2)-1}}{(n / 2)-1}-f_{n / 2}\right) \cdot 1,10
$$

Da Equação 2, os resultados foram colocados em ordem crescente $\left(f_{1} \leq f_{2} \leq f_{3} \ldots \leq f_{\mathrm{n}}\right)$, desprezando-se o maior valor da resistência em caso de número de corpos de prova ímpar, não se tomando para $\mathrm{f}_{\mathrm{c} 0 \mathrm{k}, \mathrm{k}}$ valor de resistência inferior a $f_{1}$ e nem inferior a 0,70 do valor médio das resistências. A Tabela 1 apresenta os resultados dos valores característicos e os respectivos enquadramentos das espécies nas classes de resistência da norma brasileira.

Para cada espécie de madeira foram fabricados 12 corpos de prova para cada um dos ensaios: compressão (Figura 2a), visando a determinação dos valores do $\mathrm{E}_{\mathrm{c} 0}$, tração (Figura $2 \mathrm{~b}$ ) para a obtenção dos valores do $\mathrm{E}_{\mathrm{t} 0}$, e flexão estática para a determinação dos valores do $\mathrm{E}_{\mathrm{M}}$ (Figura 3a), resultando no total de 360 determinações experimentais.

Por meio dos ensaios descritos, utilizando-se das Equações 3 a 5, foram determinados para cada amostra os valores das propriedades de rigidez $\left(\mathrm{E}_{\mathrm{c} 0}, \mathrm{E}_{\mathrm{t} 0}\right.$ e $\left.\mathrm{E}_{\mathrm{M}}\right)$.

$$
\begin{aligned}
& E_{c 0}=\frac{\sigma_{50 \%}-\sigma_{10 \%}}{\varepsilon_{50 \%}-\varepsilon_{10 \%}} \\
& E_{t 0}=\frac{\sigma_{50 \%}-\sigma_{10 \%}}{\varepsilon_{50 \%}-\varepsilon_{10 \%}} \\
& E_{M}=\frac{\left(F_{50 \%}-F_{10 \%}\right) \cdot L^{3}}{\left(v_{50 \%}-v_{10 \%}\right) \cdot 4 \cdot b \cdot h^{3}}
\end{aligned}
$$

Tabela 1 - Categorização das espécies de madeira nas classes de resistência do grupo das folhosas

\begin{tabular}{c|c|c}
\hline Espécie & $\mathbf{f}_{\mathbf{c} \mathbf{0} \mathbf{k}}(\mathbf{M P a})$ & Classe de resistência \\
\hline Cambará rosa & 25,67 & D20 \\
Cedro & 29,36 & D20 \\
Cedroarana & 24,84 & D20 \\
Canafístula & 31,83 & D30 \\
Angelim araroba & 44,24 & D40 \\
Copaíba & 45,53 & D40 \\
Louro verde & 49,14 & D40 \\
Oiticica amarela & 62,41 & D60 \\
Guaiçara & 60,10 & D60 \\
Maçaranduba & 78,20 & D60
\end{tabular}

Figura 2 - (a) Ensaio de resistência à compressão paralela às fibras; (b) ensaio de tração paralela às fibras; e (c) Corpo de prova após o ensaio de tração paralela às fibras

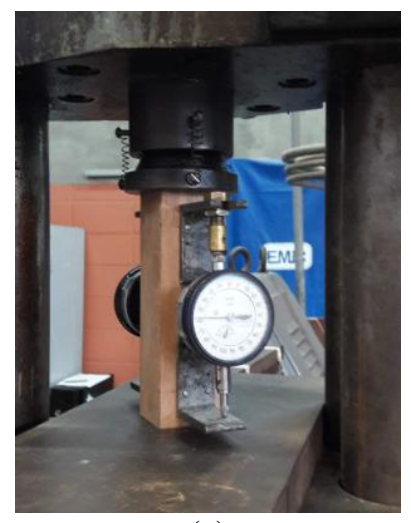

(a)

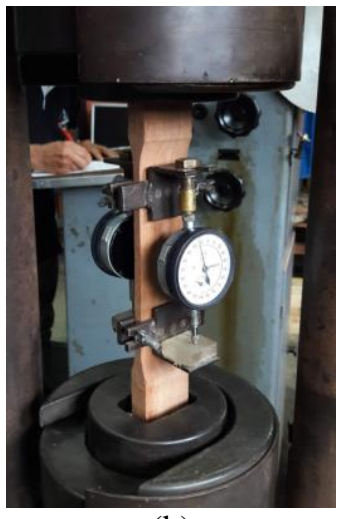

(b)

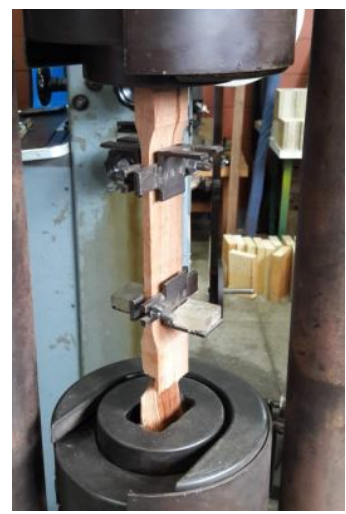

(c)

28 Almeida, J. P. B.; Couto, N. G.; Aquino, V. B. de M.; Wolenski, A. R. V.; Peixoto, R. G.; Christoforo, A. L.; Lahr, F. A. R. 
Figura 3 - (a) Ensaio de flexão estática; e (b) corpo de prova após o ensaio

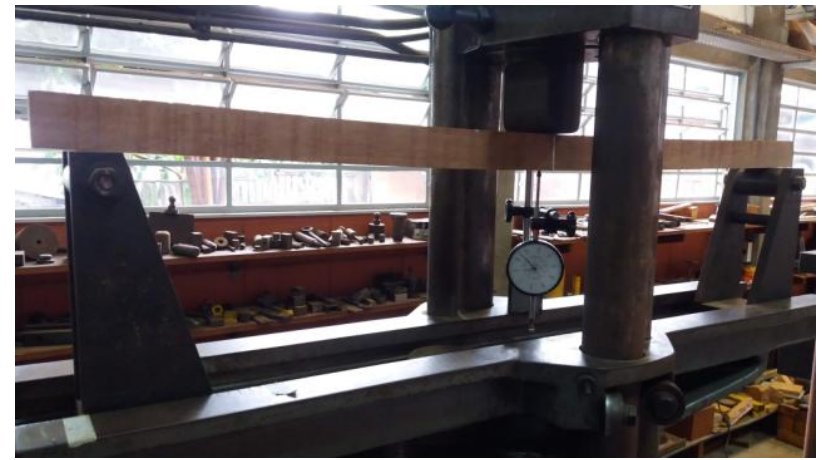

(a)

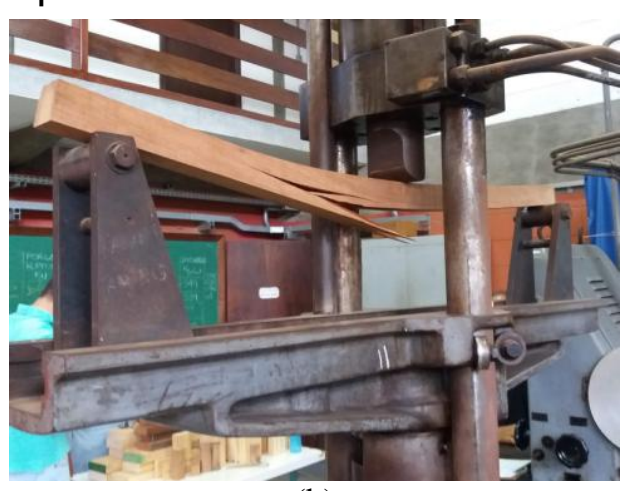

(b)

Das Equações 3 a 5, $\sigma_{50 \%}$ e $\sigma_{10 \%}$ são as tensões normais no corpo de prova, respectivamente para $50 \%$ e $10 \%$ da resistência estimada; $\varepsilon_{50 \%}$ e $\varepsilon_{10 \%}$ são as deformações específicas, respectivamente, para $50 \%$ e $10 \%$ da resistência estimada; $L$ corresponde ao vão do ensaio de flexão, $F_{50 \%}$ e $F_{10 \%}$ denotam as cargas correspondentes a $50 \%$ e $10 \%$ da carga máxima estimada; $v_{50 \%}$ e $v_{10 \%}$ referem-se aos deslocamentos no meio do vão correspondentes a $10 \%$ e $50 \%$ da carga máxima estimada; enquanto $b$ e $h$ correspondem, respectivamente, à largura e à altura da seção transversal do corpo de prova de flexão.

Inicialmente, a análise de variância (ANOVA), ao nível de 5\% de significância, foi utilizada na verificação das relações entre $\mathrm{E}_{\mathrm{t} 0}$ e $\mathrm{E}_{\mathrm{c} 0}\left(\mathrm{E}_{\mathrm{c} 0}=\mathrm{E}_{\mathrm{t} 0}\right)$ e entre $\mathrm{E}_{\mathrm{M}}$ e $\mathrm{E}_{\mathrm{c} 0}\left(\mathrm{E}_{\mathrm{M}}=0,90 \cdot \mathrm{E}_{\mathrm{c} 0}\right)$ propostas pela norma brasileira NBR 7.190 (ABNT, 1997). Acusada equivalência entre as médias (P-valor maior ou igual a 5\% - hipótese nula), isso implica a boa precisão fornecida pelas relações estabelecidas pela norma brasileira, e em caso contrário (P-valor $<5 \%$ - hipótese alternativa) novos coeficientes devem ser determinados.

No caso da não equivalência estatística entre as relações, utilizou-se do método dos mínimos quadrados (Equação 6 e 7), possibilitando determinar o coeficiente ótimo $(\lambda)$ das relações entre os módulos de elasticidade $\left(\mathrm{E}_{\mathrm{c} 0}=\lambda \cdot \mathrm{E}_{\mathrm{t} 0}\right.$ e $\left.\mathrm{E}_{\mathrm{M}}=\lambda \cdot \mathrm{E}_{\mathrm{c} 0}\right)$, com o auxílio do método de Newton comaproximação quadrática (Equação 8), ficando a convergência para solução ótima independente da escolha da estimativa inicial $\lambda_{0}$ do método iterativo.

$$
\begin{aligned}
& f(\lambda)=\frac{1}{2} \sum_{i=1}^{n}\left(E_{c 0_{i}}-\lambda \cdot E_{t 0_{i}}\right)^{2} \\
& f(\lambda)=\frac{1}{2} \sum_{i=1}^{n}\left(E_{M_{i}}-\lambda \cdot E_{c 0_{i}}\right)^{2} \\
& f(\lambda)=\frac{1}{2} \sum_{i=1}^{n}\left(E_{M_{i}}-\lambda \cdot E_{c 0_{i}}\right)^{2}
\end{aligned}
$$

\section{Resultados e discussão}

A Tabela 2 apresenta os valores médios ( $\bar{x}$ ), mínimos (Mín), máximos (Máx) e os coeficientes de variação $\left(C_{v}\right)$, das propriedades de rigidez $\left(\mathrm{E}_{\mathrm{c} 0}, \mathrm{E}_{\mathrm{t} 0}\right.$ e $\left.\mathrm{E}_{\mathrm{M}}\right)$ obtidos experimentalmente para cada uma das espécies investigadas.

Para a madeira de cambará os valores de $\mathrm{E}_{\mathrm{c} 0}(12.578 \mathrm{MPa}), \mathrm{E}_{\mathrm{t} 0}(12.636 \mathrm{MPa})$ e $\mathrm{E}_{\mathrm{M}}(12.530 \mathrm{MPa})$, determinados neste estudo, são próximos aos apresentados por Morales et al.(2018) $\left[\mathrm{E}_{\mathrm{c} 0}(12.708 \mathrm{MPa}), \mathrm{E}_{\mathrm{t} 0}\right.$ (12.509 MPa) e $\left.\mathrm{E}_{\mathrm{M}}(11.564 \mathrm{MPa})\right]$, por Lahr et al. (2016) [ $\mathrm{E}_{\mathrm{c} 0}(12.967 \mathrm{MPa}), \mathrm{E}_{\mathrm{t} 0}(12.764 \mathrm{MPa})$ e $\mathrm{E}_{\mathrm{M}}(12.376$

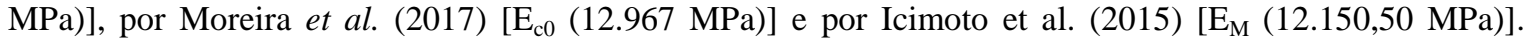
Considerando a mesma espécie, valores superiores de $\mathrm{E}_{\mathrm{c} 0}$ foram determinados por Almeida et al. (2018b), em torno de 13.000 MPa, com variações de 11.000 MPa a 15.000 MPa, e por Ferro et al. (2015) (13.867,50 $\mathrm{MPa})$. Para esta propriedade a norma brasileira apresenta valor inferior $\left[\mathrm{E}_{\mathrm{c} 0}(9.067 \mathrm{MPa})\right]$. Valores inferiores 
também foram determinados por Grobério e Lahr (2002) [ $\mathrm{E}_{\mathrm{c} 0}(8.782,8 \mathrm{MPa}), \mathrm{E}_{\mathrm{t} 0}(9.172,3 \mathrm{MPa})$ e $\mathrm{E}_{\mathrm{M}}$ (8.841,9 MPa)] e por Araújo (2007) para o $\mathrm{E}_{\mathrm{M}}(10.787 \mathrm{MPa})$.

Dias e Lahr (2004) determinaram para a madeira de cedro valores médios de 8.358 MPa, 9.851 MPa e 8.866 $\mathrm{MPa}$, correspondentes respectivamente $\mathrm{a} \mathrm{E}_{\mathrm{c} 0}, \mathrm{E}_{\mathrm{t} 0}$ e $\mathrm{E}_{\mathrm{M}}$. Tais valores são próximos aos obtidos no presente estudo $\left[\mathrm{E}_{\mathrm{c} 0}(8.270 \mathrm{MPa}), \mathrm{E}_{\mathrm{t} 0}(9.523 \mathrm{MPa})\right.$ e $\left.\mathrm{E}_{\mathrm{M}}(8.717 \mathrm{MPa})\right]$, assim como os valores de $\mathrm{E}_{\mathrm{c} 0}$ apresentados por Moreira et al. (2017) (8.354 MPa) e pela norma brasileira (8.058 MPa). Para a mesma espécie, valores superiores de $\mathrm{E}_{\mathrm{c} 0}$ foram determinados nos estudos de Ferro et al. (2015) (11.368 MPa) e Almeida et al. (2018b) (13.000 MPa), sendo que neste os valores variaram de $11.000 \mathrm{MPa}$ a $14.500 \mathrm{MPa}$.

A madeira de cedroarana apresentou valor do $\mathrm{E}_{\mathrm{M}}$ correspondente a $10.119 \mathrm{MPa}$, com variações de 8.404 $\mathrm{MPa}$ a 12.324 MPa. Gonçalez e Gonçalves (2001) determinaram valor médio de $\mathrm{E}_{\mathrm{M}}$ igual a $11.500 \mathrm{MPa}$, contemplado na faixa de valores obtidos neste estudo. $\mathrm{O} \mathrm{E}_{\mathrm{M}}$, assim como os demais valores das propriedades de rigidez obtidos $\left[\mathrm{E}_{\mathrm{c} 0}(10.394 \mathrm{MPa}), \mathrm{E}_{\mathrm{t} 0}(10.967 \mathrm{MPa})\right]$, foram condizentes aos determinados por Grobério e Lahr (2002) [ $\mathrm{E}_{\mathrm{c} 0}(10.252,4 \mathrm{MPa}), \mathrm{E}_{\mathrm{t} 0}(10.969,7 \mathrm{MPa})$ e $\left.\mathrm{E}_{\mathrm{M}}(10.032,4 \mathrm{MPa})\right]$. Para a mesma espécie, o resultado obtido do $\mathrm{E}_{\mathrm{c} 0}(10.394 \mathrm{MPa})$ diverge do encontrado por Moreira et al. (2017) (8.962 MPa).

Tabela 2 - Resultados das propriedades de rigidez das espécies de madeira estudadas

\begin{tabular}{|c|c|c|c|c|c|c|c|c|}
\hline \multirow[b]{2}{*}{$\begin{array}{l}\text { Propriedades } \\
\text { de rigidez }\end{array}$} & \multicolumn{4}{|c|}{ Cambará rosa } & \multicolumn{4}{|c|}{ Cedro } \\
\hline & $\begin{array}{c}\bar{x} \\
(\mathbf{M P a}) \\
\end{array}$ & $\begin{array}{l}C v \\
(\%) \\
\end{array}$ & $\begin{array}{c}\text { Mín } \\
\text { (MPa) }\end{array}$ & $\begin{array}{c}\text { Máx } \\
\text { (MPa) }\end{array}$ & $\begin{array}{c}\bar{x} \\
(\mathbf{M P a}) \\
\end{array}$ & $\begin{array}{l}C v \\
(\%)\end{array}$ & $\begin{array}{c}\text { Mín } \\
\text { (MPa) }\end{array}$ & $\begin{array}{c}\text { Máx } \\
\text { (MPa) }\end{array}$ \\
\hline $\mathrm{E}_{\mathrm{c} 0}$ & 12.578 & 18 & 9.635 & 16.790 & 8.270 & 14 & 6.515 & 10.915 \\
\hline $\mathrm{E}_{\mathrm{t} 0}$ & 12.636 & 14 & 10.692 & 15.728 & 9.523 & 20 & 6.743 & 13.499 \\
\hline $\mathrm{E}_{\mathrm{M}}$ & 12.530 & 8 & 10.161 & 13.978 & 8.717 & 20 & 6.591 & 12.384 \\
\hline \multirow[b]{2}{*}{$\begin{array}{l}\text { Propriedades } \\
\text { de rigidez }\end{array}$} & \multicolumn{4}{|c|}{ Cedroarana } & \multicolumn{4}{|c|}{ Canafístula } \\
\hline & $\begin{array}{c}\bar{x} \\
\text { (MPa) } \\
\end{array}$ & $\begin{array}{l}C v \\
(\%)\end{array}$ & $\begin{array}{c}\text { Mín } \\
\text { (MPa) }\end{array}$ & $\begin{array}{c}\text { Máx } \\
\text { (MPa) }\end{array}$ & $\begin{array}{c}\bar{x} \\
(\mathbf{M P a}) \\
\end{array}$ & $\begin{array}{l}C v \\
(\%)\end{array}$ & $\begin{array}{c}\text { Mín } \\
\text { (MPa) }\end{array}$ & $\begin{array}{c}\text { Máx } \\
\text { (MPa) }\end{array}$ \\
\hline $\mathrm{E}_{\mathrm{c} 0}$ & 10.394 & 13 & 8.484 & 12.913 & 14.175 & 19 & 9.882 & 17.982 \\
\hline $\mathrm{E}_{\mathrm{t} 0}$ & 10.967 & 15 & 8.113 & 13.304 & 13.665 & 17 & 9.021 & 17.034 \\
\hline $\mathrm{E}_{\mathrm{M}}$ & 10.119 & 11 & 8.404 & 12.324 & 14.326 & 16 & 10.537 & 18.293 \\
\hline \multirow[b]{2}{*}{$\begin{array}{l}\text { Propriedades } \\
\text { de rigidez }\end{array}$} & \multicolumn{4}{|c|}{ Angelim araroba } & \multicolumn{4}{|c|}{ Copaíba } \\
\hline & $\begin{array}{c}\bar{x} \\
(\mathbf{M P a})\end{array}$ & $\begin{array}{l}C v \\
(\%)\end{array}$ & $\begin{array}{c}\text { Mín } \\
\text { (MPa) }\end{array}$ & $\begin{array}{c}\text { Máx } \\
\text { (MPa) }\end{array}$ & $\begin{array}{c}\bar{x} \\
(\mathbf{M P a})\end{array}$ & $\begin{array}{l}C v \\
(\%)\end{array}$ & $\begin{array}{c}\text { Mín } \\
\text { (MPa) }\end{array}$ & $\begin{array}{c}\text { Máx } \\
\text { (MPa) }\end{array}$ \\
\hline $\mathrm{E}_{\mathrm{c} 0}$ & 12.714 & 19 & 9.850 & 18.308 & 12.662 & 17 & 9.416 & 16.018 \\
\hline $\mathrm{E}_{\mathrm{t} 0}$ & 12.371 & 16 & 9.383 & 16.093 & 13.188 & 12 & 10.591 & 15.482 \\
\hline $\mathrm{E}_{\mathrm{M}}$ & 12.633 & 22 & 8.126 & 17.392 & 12.617 & 11 & 10.164 & 14.554 \\
\hline \multirow[b]{2}{*}{$\begin{array}{l}\text { Propriedades } \\
\text { de rigidez }\end{array}$} & \multicolumn{4}{|c|}{ Louro verde } & \multicolumn{4}{|c|}{ Oiticica amarela } \\
\hline & $\begin{array}{c}\bar{x} \\
(\mathbf{M P a}) \\
\end{array}$ & $\begin{array}{l}C v \\
(\%)\end{array}$ & $\begin{array}{c}\text { Mín } \\
\text { (MPa) }\end{array}$ & $\begin{array}{c}\text { Máx } \\
\text { (MPa) }\end{array}$ & $\begin{array}{c}\bar{x} \\
(\mathbf{M P a})\end{array}$ & $\begin{array}{l}C v \\
(\%)\end{array}$ & $\begin{array}{c}\text { Mín } \\
\text { (MPa) }\end{array}$ & $\begin{array}{c}\text { Máx } \\
\text { (MPa) }\end{array}$ \\
\hline$\overline{\mathrm{E}_{\mathrm{c} 0}}$ & 14.649 & 16 & 10.577 & 18.929 & 14.277 & 9 & 11.994 & 16.889 \\
\hline $\mathrm{E}_{\mathrm{t} 0}$ & 14.026 & 17 & 11.339 & 19.467 & 14.235 & 10 & 12.466 & 16.661 \\
\hline $\mathrm{E}_{\mathrm{M}}$ & 15.178 & 21 & 9.689 & 19.487 & 14.057 & 9 & 12.281 & 16.871 \\
\hline \multirow[b]{2}{*}{$\begin{array}{l}\text { Propriedades } \\
\text { de rigidez }\end{array}$} & \multicolumn{4}{|c|}{ Guaiçara } & \multicolumn{4}{|c|}{ Maçaranduba } \\
\hline & $\begin{array}{c}\bar{x} \\
(\mathbf{M P a}) \\
\end{array}$ & $\begin{array}{l}C v \\
(\%) \\
\end{array}$ & $\begin{array}{c}\text { Mín } \\
\text { (MPa) }\end{array}$ & $\begin{array}{c}\text { Máx } \\
\text { (MPa) }\end{array}$ & $\begin{array}{c}\bar{x} \\
(\mathbf{M P a}) \\
\end{array}$ & $\begin{array}{l}C v \\
(\%) \\
\end{array}$ & $\begin{array}{c}\text { Mín } \\
\text { (MPa) }\end{array}$ & $\begin{array}{c}\text { Máx } \\
\text { (MPa) }\end{array}$ \\
\hline $\mathrm{E}_{\mathrm{c} 0}$ & 15.148 & 16 & 11.073 & 17.959 & 21.681 & 12 & 17.832 & 26.488 \\
\hline $\mathrm{E}_{\mathrm{t} 0}$ & 16.055 & 13 & 12.423 & 19.702 & 20.933 & 13 & 14.787 & 23.810 \\
\hline $\mathrm{E}_{\mathrm{M}}$ & 15.132 & 11 & 12.521 & 17.225 & 19.934 & 12 & 17.743 & 24.528 \\
\hline
\end{tabular}

30 Almeida, J. P. B.; Couto, N. G.; Aquino, V. B. de M.; Wolenski, A. R. V.; Peixoto, R. G.; Christoforo, A. L.; Lahr, F. A. $R$. 
Em relação aos valores das propriedades de rigidez da madeira de canafístula $\left[\mathrm{E}_{\mathrm{c} 0}(14.175 \mathrm{MPa}), \mathrm{E}_{\mathrm{t} 0}(13.665\right.$ $\mathrm{MPa})$ e $\mathrm{E}_{\mathrm{M}}(14.326 \mathrm{MPa})$ ], resultados próximos foram determinados por Dias e Lahr (2004) [ $\mathrm{E}_{\mathrm{c} 0}(14.613$ $\mathrm{MPa}), \mathrm{E}_{\mathrm{t} 0}(14.087 \mathrm{MPa})$ e $\mathrm{E}_{\mathrm{M}}(14.769 \mathrm{MPa})$ ] e também pela norma brasileira [ $\mathrm{E}_{\mathrm{c} 0}(14.613 \mathrm{MPa})$ ]. No que diz respeito ao $\mathrm{E}_{\mathrm{t} 0}$, o valor determinado neste estudo mostrou-se inferior ao encontrado por Faria et al. (2012) (15.281,7 MPa). Lahr et al. (2017), em estudo sobre relações entre o módulo de elasticidade longitudinal e transversal da madeira, determinaram, para a espécie de canafístula, os valores de $14.288 \mathrm{MPa}$ e $14.466 \mathrm{MPa}$ correspondentes respectivamente aos módulos de elasticidade na flexão ensaiados no plano longitudinalradial $\left[\mathrm{E}_{\mathrm{M}(\mathrm{LR})}\right]$ e no plano longitudinal-transversal $\left[\mathrm{E}_{\mathrm{M}(\mathrm{LT})}\right]$, considerando para tanto ensaios de flexão a quatro pontos. Tais valores de $\mathrm{E}_{\mathrm{M}}$ são próximos ao determinado neste estudo.

Os valores das propriedades de rigidez da madeira de angelim araroba, determinados no presente estudo [ $\mathrm{E}_{\mathrm{c} 0}$ $(12.714 \mathrm{MPa}), \mathrm{E}_{\mathrm{t} 0}(12.371 \mathrm{MPa})$ e $\left.\mathrm{E}_{\mathrm{M}}(12.633 \mathrm{MPa})\right]$, mostraram-se próximos aos valores encontrados por Grobério e Lahr (2002) [ $\mathrm{E}_{\mathrm{c} 0}(12.525,4 \mathrm{MPa}), \mathrm{E}_{\mathrm{t} 0}(11.885,4 \mathrm{MPa})$ e $\left.\mathrm{E}_{\mathrm{M}}(11.943,3 \mathrm{MPa})\right]$ e por Dias e Lahr (2004) $\left[\mathrm{E}_{\mathrm{c} 0}(12.587 \mathrm{MPa}), \mathrm{E}_{\mathrm{t} 0}(11.661 \mathrm{MPa})\right.$ e $\left.\mathrm{E}_{\mathrm{M}}(11.457 \mathrm{MPa})\right]$. No que diz respeito ao $\mathrm{E}_{\mathrm{c} 0}$, o valor determinado é próximo ao apresentado pela norma brasileira (12.876 MPa).

Em relação a madeira de copaíba, os valores de $\mathrm{E}_{\mathrm{c} 0}(12.662 \mathrm{MPa}), \mathrm{E}_{\mathrm{t} 0}(13.188 \mathrm{MPa})$ e $\mathrm{E}_{\mathrm{M}}(12.617 \mathrm{MPa})$, determinados neste estudo, foram próximos aos encontrados por Araújo (2007) [ $\mathrm{E}_{\mathrm{M}}$ (12.062 MPa)], por

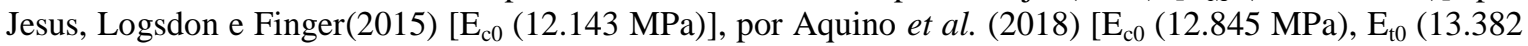
$\mathrm{MPa})$ e $\mathrm{E}_{\mathrm{M}}(12.440 \mathrm{MPa})$ ] e inferiores aos obtidos por Dias e Lahr (2004) [ $\mathrm{E}_{\mathrm{c} 0}$ (14.012 MPa), $\mathrm{E}_{\mathrm{t} 0}(14.627$ $\mathrm{MPa})$ e $\left.\mathrm{E}_{\mathrm{M}}(13.572 \mathrm{MPa})\right]$. Quanto ao $\mathrm{E}_{\mathrm{t} 0}$, o valor determinado, com variações de $10.591 \mathrm{MPa}$ a 15.482 $\mathrm{MPa}$, foi superior ao encontrado por Faria et al. (2012) (11.957,7 MPa).

Para a madeira de louro verde, os valores de $\mathrm{E}_{\mathrm{c} 0}(14.649 \mathrm{MPa}), \mathrm{E}_{\mathrm{t} 0}(14.026 \mathrm{MPa})$ e $\mathrm{E}_{\mathrm{M}}(15.178 \mathrm{MPa})$, determinados neste estudo, foram superiores aos encontrados por Dias e Lahr (2004) [ $\mathrm{E}_{\mathrm{c} 0}(13.536 \mathrm{MPa}), \mathrm{E}_{\mathrm{t} 0}$ $(12.851 \mathrm{MPa})$ e $\left.\mathrm{E}_{\mathrm{M}}(13.556 \mathrm{MPa})\right]$ e por Araújo (2007) $\left[\mathrm{E}_{\mathrm{M}}(14.024 \mathrm{MPa})\right]$. No que diz respeito ao $\mathrm{E}_{\mathrm{c} 0}$, a norma brasileiraapresenta valor próximo (14.185 MPa).

Em relação às propriedades de rigidez da madeira de oiticica amarela, o $\mathrm{E}_{\mathrm{c} 0}$ apresentou valor de 14.277 $\mathrm{MPa}$, com mínimo e máximo correspondentes respectivamente a $11.994 \mathrm{MPa}$ e $16.889 \mathrm{MPa}$. Para a mesma propriedade, Jesuset al.(2015) determinaram valor superior (17.512 $\mathrm{MPa})$, enquanto a norma brasileira apresenta valor próximo (14.719 $\mathrm{MPa}$ ). No que diz respeito ao $\mathrm{E}_{\mathrm{M}}$, o valor determinado (14.057 $\mathrm{MPa}$ ) mostrou-se próximo ao definido por Dias e Lahr (2004) (14.491 MPa) e superior ao encontrado por Araújo (2007) $(12.160 \mathrm{MPa})$. O resultado do $\mathrm{E}_{\mathrm{t} 0}(14.235 \mathrm{MPa})$ foi condizente ao valor de $14.675 \mathrm{MPa}$ apresentado por Dias e Lahr (2004).

Os valores das propriedades de rigidez para a madeira de Guaiçara, obtidos no presente estudo [ $\mathrm{E}_{\mathrm{c} 0}(15.148$ $\mathrm{MPa}), \mathrm{E}_{\mathrm{t} 0}(16.055 \mathrm{MPa})$ e $\mathrm{E}_{\mathrm{M}}(15.132 \mathrm{MPa})$ ], mostraram-se próximos aos encontrados por Grobério e Lahr (2002) para $\mathrm{E}_{\mathrm{c} 0}(15.301,7 \mathrm{MPa}), \mathrm{E}_{\mathrm{t} 0}(16.304,7 \mathrm{MPa})$ e $\mathrm{E}_{\mathrm{M}}(15.126,4 \mathrm{MPa})$ e por Moreira et al. (2017) para $\mathrm{E}_{\mathrm{c} 0}(15.301 \mathrm{MPa})$. Para a mesma espécie, valores inferiores foram apresentados por Dias e Lahr (2004) [ $\mathrm{E}_{\mathrm{c} 0}$ $(14.027 \mathrm{MPa}), \mathrm{E}_{\mathrm{t} 0}(14.946 \mathrm{MPa})$ e $\left.\mathrm{E}_{\mathrm{M}}(13.866 \mathrm{MPa})\right]$ e pela norma brasileira [ $\left.\mathrm{E}_{\mathrm{c} 0}(14.624 \mathrm{MPa})\right]$.

Para a madeira de maçaranduba, os valores determinados neste estudo [ $\mathrm{E}_{\mathrm{c} 0}(21.681 \mathrm{MPa}), \mathrm{E}_{\mathrm{t} 0}(20.933 \mathrm{MPa})$ e $\left.\mathrm{E}_{\mathrm{M}}(19.934 \mathrm{MPa})\right]$ mostraram-se próximos aos determinadospor Dias e Lahr (2004) [ $\mathrm{E}_{\mathrm{c} 0}(21.900 \mathrm{MPa}), \mathrm{E}_{\mathrm{t} 0}$ (20.267 $\mathrm{MPa}$ e $\left.\mathrm{E}_{\mathrm{M}}(18.184 \mathrm{MPa})\right]$. Para a mesma espécie, a norma brasileira apresenta valor próximo de $\mathrm{E}_{\mathrm{c} 0}$ (22.733 MPa), enquanto valores inferiores depropriedades de rigidez foram determinados por Faria et al. (2012) (17.755 MPa) e por Jesus et al.(2015) (18.620 MPa), para $\mathrm{E}_{\mathrm{t} 0}$ e $\mathrm{E}_{\mathrm{c} 0}$, respectivamente.

As comparações das propriedades apresentadas na Tabela 2 com os valores determinados na literatura validam os resultados obtidos neste trabalho. Ressalta-se, contudo, que pequenas dispersões nos resultados podem ser justificadas, em partes, pela forma como os ensaios experimentais são executados, pelo modelo de cálculo adotado para determinação dos resultados e pela variabilidade intrínseca do material.

Nas Tabelas 3 e 4 são apresentados os resultados da ANOVA das relações $E_{c 0}=E_{t 0}$ e $E_{M}=0,90 \cdot E_{c 0}$, respectivamente.

Da Tabela 3, verifica-se que a média dos grupos $\mathrm{E}_{\mathrm{c} 0}$ e $\mathrm{E}_{\mathrm{t} 0}$ são estatisticamente equivalentes (P-valor $\left.\geq 0,05\right)$, $\mathrm{o}$ que implica a boa precisão da equação $\mathrm{E}_{\mathrm{c} 0}=\mathrm{E}_{\mathrm{t} 0}$ proposta pela norma brasileira. Por outro lado, a ANOVA acusou diferença significativa nas médias entre $\mathrm{E}_{\mathrm{M}}$ e $0,90 \cdot \mathrm{E}_{\mathrm{c} 0}$ (P-valor $<0,05$ - Tabela 4), o que implica que o coeficiente de 0,90 da equação $\mathrm{E}_{\mathrm{M}}=0,90 \cdot \mathrm{E}_{\mathrm{c} 0} \mathrm{não}$ se apresenta como estimativa precisa na obtenção do módulo de elasticidade na flexão estática. 
Constatada a não equivalência entre $\mathrm{E}_{\mathrm{M}}$ e $0,90 \cdot \mathrm{E}_{\mathrm{c} 0}$, o método dos mínimos quadrados (Equação 7 e 8 ) foi utilizado para a determinação do coeficiente ótimo $(\lambda)$ da relação entre $E_{M}$ e $E_{c 0}\left(E_{M}=\lambda \cdot E_{c 0}\right)$. A Tabela 5 apresenta o resultado de $\lambda$ considerando cada espécie individualmente e o conjunto de todas as espécies estudadas.

O coeficiente ótimo obtido para o conjunto envolvendo todas as espécies apresentado na Tabela 5 corresponde a 0,98 , sendo $8,89 \%$ superior ao atual valor de 0,90 apresentado pela normativa brasileira. A Equação 9 apresenta a relação de equivalência entre o $\mathrm{E}_{\mathrm{M}}$ e $\mathrm{E}_{\mathrm{c} 0}$ obtida neste estudo.

$$
E_{M}=0,98 \cdot E_{c 0}
$$

Tabela 3 - Resultados da ANOVA (5\% de significância) para a relação $E_{c 0}=E_{t 0}$

\begin{tabular}{c|c|c|c|c|c}
\hline Fonte & GL & SQ $(\mathbf{A j .})$ & QM (Aj.) & Valor $\mathbf{F}$ & Valor-P \\
\hline $\mathrm{E}_{\mathrm{c} 0}=\mathrm{E}_{\mathrm{t} 0}$ & 1 & 60506 & 60506 & 0,00 & 0,948 \\
\hline Erro & 238 & 3388077580 & 14235620 & & \\
\hline Total & 239 & 3388138086 & & & \\
\hline
\end{tabular}

Nota: Legenda:

GL - graus de liberdade;

SQ (Aj.) - soma dos quadrados ajustados; $e$

$Q M(A j)$ - média dos quadrados ajustados.

Tabela 4 - Resultados da ANOVA (5\% de significância) para a relação $E_{M}=0,90 \cdot E_{c 0}$

\begin{tabular}{c|c|c|c|c|c}
\hline Fonte & GL & SQ (Aj.) & QM (Aj.) & Valor $\mathbf{F}$ & Valor-P \\
\hline $\mathrm{E}_{\mathrm{M}}=0,90 \cdot \mathrm{E}_{\mathrm{c} 0}$ & 1 & 81761550 & 81761550 & 6,48 & 0,012 \\
Erro & 238 & 3001462068 & 12611185 & & \\
\hline Total & 239 & 3083223618 & & & \\
\hline
\end{tabular}

Nota: Legenda:

GL - graus de liberdade;

SQ (Aj.) - soma dos quadrados ajustados; $e$

$Q M(A j)$ - média dos quadrados ajustados.

Tabela 5 - Resultado do coeficiente ótimo $(\lambda)$ da relação entre $E_{M}$ e $E_{c o}$ para cada espécie e para o conjunto de todas as espécies estudadas

\begin{tabular}{c|c}
\hline Espécie & $\boldsymbol{\lambda}\left[\mathbf{E}_{\mathbf{M}}=\boldsymbol{\lambda} \cdot \mathbf{E}_{\mathbf{c} 0}\right]$ \\
\hline Cambará rosa & 1,00 \\
Cedro & 1,05 \\
Cedroarana & 0,97 \\
Canafístula & 1,01 \\
Angelim araroba & 0,99 \\
Copaíba & 1,00 \\
Louro verde & 1,04 \\
Oiticica amarela & 0,98 \\
Guaiçara & 1,00 \\
Maçaranduba & 0,92 \\
\hline Todas as espécies & 0,98 \\
\hline
\end{tabular}

32 Almeida, J. P. B.; Couto, N. G.; Aquino, V. B. de M.; Wolenski, A. R. V.; Peixoto, R. G.; Christoforo, A. L.; Lahr, F. A. R. 


\section{Conclusão}

Este estudo contribui para ampliar o conhecimento quanto à relação entre propriedades de rigidez das madeiras folhosas, subsidiando a tomada de decisão sobre os parâmetros normativos a serem adotados. Considerando os resultados obtidos, pôde-se concluir:

(a) a ANOVA acusou equivalência estatística entre $\mathrm{E}_{\mathrm{c} 0}$ e $\mathrm{E}_{\mathrm{t} 0}$, evidenciando a boa precisão da equação $\mathrm{E}_{\mathrm{c} 0}=$ $\mathrm{E}_{\mathrm{t} 0}$. Cabe ressaltar que esta conclusão é pertinente apenas para as madeiras folhosas, haja vista que a equação entre $\mathrm{E}_{\mathrm{c} 0}$ e $\mathrm{E}_{\mathrm{t} 0}$ prescrita pela norma brasileira não faz distinção entre folhosas e coníferas;

(b) pela ANOVA, a média dos grupos $\mathrm{E}_{\mathrm{M}}$ e $0,90 \cdot \mathrm{E}_{\mathrm{c} 0}$ apresentaram diferença significativa, implicando que o coeficiente 0,90 da equação $\mathrm{E}_{\mathrm{M}}=0,90 \cdot \mathrm{E}_{\mathrm{c} 0}$ não conduz a obtenção do $\mathrm{E}_{\mathrm{M}}$;

(c) o valor do coeficiente ótimo $(\lambda)$ obtido no presente estudo para a relação entre $\mathrm{E}_{\mathrm{M}}$ e $\mathrm{E}_{\mathrm{c} 0}$ corresponde a 0,98, sendo 8,89\% superior ao estabelecido pela NBR 7190 (Item 6.3.4 (ABNT, 1997));

(d) nas verificações do estado limite de utilização de projetos de estrutura de madeira, a adoção dos valores de $\mathrm{E}_{\mathrm{c} 0}$ determinados a partir de $\mathrm{E}_{\mathrm{M}}$, por meio da Equação 9, conduzem a deslocamentos verticais maiores em relação aos calculados adotando-se os valores de $\mathrm{E}_{\mathrm{c} 0}$ obtidos pela equação $\mathrm{E}_{\mathrm{M}}=0,90 \cdot \mathrm{E}_{\mathrm{c} 0}$. Desse modo, $\mathrm{o}$ emprego da equação $\mathrm{E}_{\mathrm{M}}=0,98 \cdot \mathrm{E}_{\mathrm{c} 0}$ conduz a elementos estruturais de maior inércia, e, portanto, a favor da segurança; e

(e) para a revisão desta normativa, sugere-se o ajuste do coeficiente da equação $E_{M}=0,90 \cdot E_{c 0}$ conforme apresentado na Equação 9, com o intuito de assegurar projetos estruturais mais precisos, visto a importância do módulo de elasticidade no dimensionamento de estruturas de madeira.

\section{Referências}

ALMEIDA, A. S. et al. Evaluation of Stiffness in compression perpendicular to grain of Brazilian tropical wood species. Current Journal of Applied Science and Technology, v. 28, n. 5, p.1-7, $2018 \mathrm{~b}$.

ALMEIDA, D. H. et al. Tenacidade da madeira como função da densidade aparente. Revista Árvore, Viçosa, v. 38, n. 1, p. 203-207, 2014.

ALMEIDA, T. H. et al. Effect of service temperature on shear strength of Pinus wood for roof structures. Acta Scientiarum. Technology, v. 40, p. 2-6, 2018a.

AQUINO, V. B. M. et al. Physical and mechanical characterization of Copaifera sp. wood specie. International Journal of Materials Engineering, v. 8, n. 3, p. 55-58, 2018.

ARAUJO, H. J. B. Relações funcionais entre propriedades físicas e mecânicas de madeiras tropicais brasileiras. Floresta, Curitiba, v. 37, n. 3, set/dez. 2007.

ARAUJO, V. A. et al. Classification of wooden housing building systems. BioResources, v. 11, n. 3, p. 7889-7901, 2016.

ARAUJO, V.A. et al. Difficulties of wooden housing production sector in Brazil. Wood Material Science \& Engineering, p. 1-10, 2018.

ASSOCIAÇÃO BRASILEIRA DE NORMAS TÉCNICAS. MB-26 (NBR 6230): ensaios físicos e mecânicos de madeira. Rio de Janeiro, 1940.

ASSOCIAÇÃO BRASILEIRA DE NORMAS TÉCNICAS. NBR 7190: projeto de estruturas de madeira. Rio de Janeiro, 1997.

BEECH, E. et al. GlobalTreeSearch: the first complete global database of tree species and country distributions. Journal of Sustainable Foresty, v. 36, p. 454-489, 2017.

CALIL Jr., C.; DIAS, A. A. Utilização da madeira em construções rurais. Revista Brasileira de Engenharia Agrícola e Ambiental, Campina Grande, v. 1, p. 71-77, 1997.

CHRISTOFORO, A. L. et al. Characterization of tropical wood species for use in civil constructions. Journal of Civil Engineering Research, v. 3, n. 3, p. 98-103, 2013a.

CHRISTOFORO, A. L. et al. Metodologia para o cálculo dos módulos de elasticidade longitudinal e transversal em vigas de madeira de dimensões estruturais. Ciência Rural, Santa Maria, v. 43, n. 4, p. 610$615,2013 b$ 
DIAS, F. M.; LAHR, F. A. R. Estimativa de propriedades de resistência e rigidez da madeira através da densidade aparente. Scientia Florestalis, Piracicaba, v. 65, p. 102-113, jun. 2004.

FARIA, O. B. et al. Influence of wood moisture content on modulus of elasticity on tension parallel to the grain of Brazilian species. European International Journal of Science and Technology, v. 1, n. 2, p. 1122, 2012.

FERRO, F. S. et al.. Influência da posição dos instrumentos de medida na determinação do módulo de elasticidade da madeira na compressão paralela às fibras $\left(\mathrm{E}_{\mathrm{c} 0}\right)$. Revista Árvore, Viçosa, v. 39, n. 4, p. 743$749,2015$.

GONÇALEZ, J. C.; GONÇALVES, D. M. Valorização de duas espécies de madeira Cedrelinga catenaeformis e Enterolobium shomburgkii para a indústria madereira. Brasil Florestal, v. 70, p. 69-74, jun. 2001.

GROBÉRIO, M. P.; LAHR, F. A. R. Indicações para o emprego da madeira de espécies tropicais do Brasil. Revista Madeira: arquitetura e engenharia, v. 3, n. 8, 2002.

HURMEKOSKI, E.; JONSSON, R.; NORD, T. Context, drivers, and future potential for wood-frame multistory construction in Europe. Technological Forecasting \& Social Change, v. 99, p. 181-196, 2015.

ICIMOTO, F. H. et al. Influence of specimen orientation on determination of elasticity in static bending. Maderas. Ciencia y Tecnología, v. 17, n. 5, p. 229-238, 2015.

JESUS, J. M. H.; LOGSDON, N. B.; FINGER, Z. Classes de resistência de algumas madeiras de Mato Grosso. Engineering and Science, v. 1, n. 3, 2015.

LAHR, F. A. R. et al. Full Characterization of Erisma uncinatum warm wood specie. International Journal of Materials Engineering, v. 6, n. 5, p. 147-150, 2016.

LAHR, F. A. R. et al. Shear and longitudinal modulus of elasticity in wood: relations based on static bending tests. Acta Scientiarum. Technology, v. 39, n.4, p. 433-437, oct./dec. 2017.

LAHR, F. A. R. Sobre a determinação de propriedades de elasticidade da madeira. São Carlos, 1983. 221 f. Tese (Doutorado em Engenharia de Estruturas) - Escola de Engenharia, Universidade de São Paulo, São Carlos, 1983.

MAHAPATRA, K.; GUSTAVSSON, L.; HEMSTRÖM, K. Multi-storey wood-frame buildings in Germany, Sweden and the UK. Construction Innovation, v. 12, p. 62-85, 2012.

MORALES, E. A. M. et al. Evaluation of the number of load cycles to determine some wood stiffness properties. Current Journal of Applied Science and Technology, v. 29, n. 1, p. 1-7, 2018.

MOREIRA, A. P. et al. Toughness and impact strength in dynamic bending of wood as a function of the modulus of elasticity and the strength in compression to the grain. International Journal of Materials Engineering, v. 7, n. 4, p. 61-67, 2017.

PEDRESCHI, R.; GOMES, F. C.; MENDES, L. M. Avaliação do desempenho da madeira na habitação utilizando abordagens de sistemas. Cerne, Lavras, v. 11, n. 3, p. 283-293, jul./set. 2005.

RAMAGE, M. H. et al. The wood from the tress: The use of timber in construction. Renewable and Sustainable Energy Reviews, v. 68, p. 333-359, 2017.

VIDAL, J. M. et al. Preservação de madeiras no Brasil: histórico, cenário atual e tendências. Ciência Florestal, Santa Maria, v. 25, n. 1, p. 257-271, 2015.

WIERUSZEWSKI, M.; MAZELA, B. Cross Laminated Timber (CLT) as an alternative form of construction wood. Drvna industrija, v. 68, n. 4, p. 359-367, 2017.

ZANGIÁCOMO, A. L.; CHRISTOFORO, A. L.; LAHR, F. A. R. Módulo de elasticidade aparente em vigas roliças estruturais de madeira Pinus elliottii. Ambiente Construído, Porto Alegre, v. 14, n. 1, p. 7-13, jan./mar. 2014.

34 Almeida, J. P. B.; Couto, N. G.; Aquino, V. B. de M.; Wolenski, A. R. V.; Peixoto, R. G.; Christoforo, A. L.; Lahr, F. A. R. 


\title{
Errata
}

No artigo "Relações entre propriedades de rigidez para distintas solicitações mecânicas visando projetos de estruturas de madeira", com número de DOI: <http://dx.doi.org/10.1590/s1678-86212020000200385>, publicado no periódico Ambiente Construído, 20(2):25-35.

Nas páginas25 e 35:

Onde se lia:

"Rodrigo Peixoto Guerra"

Leia-se:

"Rodrigo Guerra Peixoto"

Na página 271 e nas páginas pares:

Onde se lia:

"GUERRA, R. P."

Leia-se:

"PEIXOTO, R. G."

\section{João Paulo Boff Almeida}

Departamento de Engenharia Civil | Universidade Federal de São Carlos | Rodovia Washington Luís, km 235, s/n, Jardim Guanabara | São Carlos - SP - Brasil | CEP 13565-905 | Tel.: (16) 3351-8262 | E-mail: boff.joaopaulo@gmail.com

\section{Natalia Garcia Couto}

Departamento de Engenharia Civil | Universidade Federal de São Carlos | E-mail: natygcouto@gmail.com

\section{Vinicius Borges de Moura Aquino}

Faculdade de Tecnologia | Universidade Federal do Sul e Sudeste do Pará | Rua Geraldo Ramalho, s/n, Centro | Santana do Araguaia - PA - Brasil | CEP 68560-000 | Tel.: (94) 2101-5936 | E-mail: aquino.vini@hotmail.com

\section{Anderson Renato Vobornik Wolenski}

Departamento de Engenharia Civil | Instituto Federal de Santa Catarina | Av. Aloísio Stoffel, 1271, Jardim Alvorada | São Carlos - SC Brasil | CEP 89885-000 | Tel.: (49) 3325-4149 | E-mail: anderson.wolenski@ifsc.edu.br

\section{Rodrigo Guerra Peixoto}

Departamento de Engenharia de Estruturas | Universidade Federal de Minas Gerais | Av. Antonio Carlos, 6627, Pampulha | Belo Horizonte - MG - Brasil | CEP 31270-901 | Tel.: (31) 3409-1980 | E-mail: rodrigo.peixoto@dees.ufmg.br

\section{André Luis Christoforo}

Departamento de Engenharia Civil | Universidade Federal de São Carlos | Rodovia Washington Luís, km 235, s/n, Jardim Guanabara | São Carlos - SP- Brasil | CEP 13565-905 | Tel.: (16) 3306-6828 | E-mail: christoforoal@yahoo.com.br

Francisco Antonio Rocco Lahr

Departamento de Engenharia Civil | Universidade de São Paulo | Av. Trabalhador São Carlense, 400, Centro | CEP 13566-590 | Tel.: (16) 3373-8206 | E-mail: frocco@sc.usp.br

\author{
Ambiente Construído \\ Revista da Associação Nacional de Tecnologia do Ambiente Construído \\ Av. Osvaldo Aranha, $99-3^{\circ}$ andar, Centro \\ Porto Alegre - RS - Brasil \\ CEP 90035-190 \\ Telefone: +55 (51) 3308-4084 \\ Fax: +55 (51) 3308-4054 \\ www.seer.ufrgs.br/ambienteconstruido \\ E-mail: ambienteconstruido@ufrgs.br
}

(c) (i) This is an open-access article distributed under the terms of the Creative Commons Attribution License. 